\section{AB0801 \\ RELIABILITY OF A NOVEL ULTRASONOGRAPHIC SCALE FOR ACTIVITY OF KNEE OSTEOARTHRITIS}

Mohammed A. Mortada, Ghada Dawa, Amany Ebaid, Lobna Kotb, Youmna A. Amer. Faculty of Medicine. Zagazig University, Rheumatology and Rehabilitation, Zagazig, Egypt

Background: Knee osteoarthritis

Objectives: To assess the reliability of a novel ultrasonographic scale of activity in knee osteoarthritis (OA)

Methods: A cross-sectional observational study included 110 patients with knee pain who fulfilled the American College of Rheumatology (ACR) criteria for knee osteoarthritis $(\mathrm{OA})$. All patients were subjected to clinical assessment WOMAC scale (Western Ontario and McMaster Universities Index of Osteoarthritis and global visual analog scale) and functional assessment using health assessment questionnaire (HAQ). Ultrasonographic assessment of activity was done By 3 rheumatologists with different levels of experience in musculoskeletal Ultrasonography (1-12 years). Ultrasonographic assessments were done according to (MOAKA scale) that was proposed by the first author (table 1).

Abstract AB0801 Table 1. Mortada OsteoaArthritis Knee Activity score (MOAKA score)

\begin{tabular}{llc}
\hline Domain & Description & Score \\
\hline Severity of knee & 6 grades according to severity scale published by & Grade 0:
\end{tabular}

$\mathrm{OA} \quad$ Mortada et al 2016 (1)

0

Grade 1:

1

Grade2a:

2

Grade 2b:

3

Grade 3:

4

Grade 4:

5

Effusion

4 grades

Grade 0:no effusion

Grade 1: Mild effusion

Grade 2: moderate effusion

Grade 3: severe effusion

Grade 0:

Grade 1:

1

Grade 2:

2

Grade 3: 3

Synovitis 4 grades using the combined EULAR/OMERACT score of Grade 0: grey scale synovitis and Doppler activity

Pes Anserine tendonitis/bursitis

3 grades

Grade 0:normal

Grade 1: mild inflammation

Grade 2: severe inflammation

Backer cyst

3 grades;

Grade 0: normal no cyst

Grade 1: small and simple cyst

Grade 2: large and/or complicated cyst

Total scores

Sum of scores of all domains

0

Grade 1:

1

Grade 2:

2

Grade 3:

Grade 0:

0

Grade 1:

1

Grade 2:

Grade 0:

0

Grade 1:

1

Grade 2:

2

$0-15$

Results: There were high kappa values both in intraobserver and interobserver evaluation of activity of knee OA using the proposed (MOAKA) ultrasonographic scale ( 0.85 and 0.75 respectively).

There were positive correlations between MOAKA score and all WOMAC subscales (pain, stiffness and function) $(r=0.4, P=0.02, \quad r=0.35, P=0.001$ and $r=0.4, P=0.01$ ) respectively.

Also there were a strong positive correlation between MOAKA scale and both (VAS and HAQ) $(r=0.86, P=0.001$ and $r=0.71, P=0.001)$

Conclusion: US can reliably detect the activity of Knee OA. Good agreement was found between the proposed US grading scale and WOMAC \& HAQ scores. MOAKA US scale is simple and reliable.

Disclosure of Interests: None declared

DOI: 10.1136/annrheumdis-2019-eular.1727
$\mathrm{AB} 0802$

CYTOKINE PROFILE IN SYNOVIAL FLUID FROM PATIENTS WITH OSTEOARTHRITIS WITH OR WITHOUT CALCIUM CRYSTALS

Francesca Oliviero ${ }^{1}$, Paola Galozzi ${ }^{1}$, Marta Favero ${ }^{1}$, Davide Tietto ${ }^{2}$,

Mariagrazia Lorenzin ${ }^{1}$, Augusta Ortolan ${ }^{1}$, Leonardo Punzi ${ }^{3}$, Anna Scanu ${ }^{1}$,

Roberta Ramonda'. ' University of Padova, Department of Medicine, Padova, Italy;

${ }^{2}$ Fisiored - Fisioterapia e Riabilitazione, Padova, Italy, ${ }^{3}$ Centre for Gout and

Metabolic Bone and Joint Diseases, Rheumatology, SS Giovanni and Paolo,

Venezia, Italy

Background: The most important pathogenetic calcium crystals that can be found in synovial fluid (SF) are calcium pyrophosphate (CPP) and basic calcium phosphate (BCP) crystals. We and others have demonstrated that a good portion of patients with osteoarthritis $(\mathrm{OA})$ have these type of crystals in their SF and that they are associated with a higher inflammatory state independently from the disease severity [1].

Objectives: The aim of this study was to investigate the levels of IL-1b, IL-8, IL-6, IL-10, CCL2 and OSMr in the SF of patients affected with OA considering the presence of CPP crystals and the positivity to the alizarin red test (a non-specific test for BCP crystals). A sub-analysis has been conducted subdividing patients according to their Kellgren-Lawrence (K-L) radiographic score.

Methods: Synovial fluid was collected from 69 OA consecutive patients diagnosed according to the EULAR criteria. Forty patients were negative to (CPP-) and 29 positive to (CPP+) CPP crystals. A standard analysis was performed for each sample including, white blood cell (WBC) count, differential cell count, crystal search under polarized light microscopy and alizarin red test. The cytokines were measured after appropriate dilutions by ELISA (Thermofisher) and expressed as $\mathrm{pg} / \mathrm{ml}$. The Mann-Whitney test was used to investigate differences between the groups of patients positive and negative to CPP; the Spearman rank test was used for correlations, while the Kruskal-Wallis to compare the groups according to the $\mathrm{K}$ $\mathrm{L}$ score.

Results: With respect to the group of CPP-, the group of CPP+ patients had higher levels of WBC count $(251.7 \pm 199.3$ vs $176.3 \pm 161.8$ cells $/ \mathrm{mm} 3$ $\mathrm{p}=0.004), \quad \mathrm{PMN} \% \quad(5 \pm 7.1$ vs $0.44 \pm 1.54 ; \mathrm{p}<0.0001), \quad \mathrm{IL}-1 \mathrm{~b} \quad(8.92 \pm 6.29$ vs $4.63 \pm 12.2 ; \mathrm{p}<0.0001)$ and IL-8 $(53.73 \pm 45.83$ vs $26.20 \pm 35.94 ; \mathrm{p}=0.0003)$. No differences were instead observed for the other cytokines considered. We did not find any differences subdividing patients according to the SF positivity to the alizarin red staining. Some correlations were observed in the whole group of patients between IL-1 and IL-8 $(\mathrm{p}=0.004)$, IL-1 and PMN $(p=0.0006), I L-8$ and WBC $(p=0.001), I L-8$ and PMN $(p<0.0001)$, IL10 and IL-8 $(\mathrm{p}=0.04), \mathrm{LL}-10$ and WBC $(\mathrm{p}=0.014)$, CCL2 and IL-6 (0.006). Although no differences have been found analysing the variables in the groups subdivided by the K-L scores, all the cytokines showed higher levels in patients with a K-L score equal to 2 .

Conclusion: Although associated with higher inflammatory SF indices and IL-1 and IL-8 levels, calcium crystals still play an undefined role in OA. More studies are warranted to evaluate if patients with calcium crystals in their SF might need a more specific treatment.

\section{REFERENCE}

[1] Frallonardo P, Ramonda R, Peruzzo L, Scanu A, Galozzi P, Tauro L, et al. Basic calcium phosphate and pyrophosphate crystals in early and late osteoarthritis: relationship with clinical indices and inflammation. Clin Rheumatol. 2018 Jun 7.

Disclosure of Interests: Francesca Oliviero: None declared, Paola Galozzi: None declared, Marta Favero: None declared, Davide Tietto: None declared, Mariagrazia Lorenzin: None declared, Augusta Ortolan: None declared, Leonardo Punzi Consultant for: BMS, Fidia, Grunenthal, Menarini, Speakers bureau: BMS, Fidia, Grunenthal, Menarini, Anna Scanu: None declared, Roberta Ramonda: None declared DOI: 10.1136/annrheumdis-2019-eular.6257

\section{AB0803 SYNOVIAL INTERLEUKIN-8 LEVELS ARE ASSOCIATED TO RADIOGRAPHIC SEVERITY OF KNEE OSTEOARTHRITIS. A CROSS-SECTIONAL STUDY}

Silvia Garcia-Cirera, Joan Calvet, Cristobal Orellana, Noemí Navarro, Maria Garcia Manrique de Lara, Jordi Gratacos-Masmitja. Hospital Universitari Parc Tauli Sabadell, Rheumatology, Sabadell, Spain

Background: Radiographic severity is used in routine clinical practice to evaluate knee osteoarthritis (KOA) structural damage. There are no conclusive studies on the associations between synovial inflammatory markers and the different features of radiographic damage in KOA. 